\title{
Avaliação dos valores hematológicos em cavalos (Equus caballus) da raça Puro-Sangue-Inglês (PSI) submetidos a teste de esforço em esteira ergométrica
}

\section{Evaluation of hematologic values in thoroughbred (PSI) horses (Equus caballus) submitted to effort tests on treadmill}

\author{
Rosalie Joslin Kowal, ${ }^{*}$ Nádia Regina Pereira Almosny,, ${ }^{* *}$ Bianca Cascardo, ${ }^{* * *}$ \\ Ricardo Peixoto Summa, ${ }^{* * *}$ Leopoldo José Cury****
}

\begin{abstract}
Resumo
O Puro-Sangue-Inglês (PSI) encontra-se difundido em todo o Brasil e dada à falta de dados sobre a fisiologia do exercício destes animais, torna-se importante a realização de testes de esforço sob algumas condições características visando a obter informações mais precisas e adequadas quando esses cavalos são submetidos a esforços constantes. Por muitos anos medidas como hematócrito e concentração de hemoglobina foram usadas para avaliar a capacidade atlética. Outros parâmetros, porém, mostraram-se de grande valia na determinação de performance. Logo, foram utilizados 20 animais em fase de treinamento no Jockey Club Brasileiro, com idade entre 2 e 6 anos, machos e fêmeas, submetidos a testes de esforço em esteira ergométrica visando avaliar dados hematológicos (contagem total de células vermelhas e brancas, volume globular (VG), concentração de hemoglobina e valor das proteínas plasmáticas). Os valores médios variaram nos tempos estudados e foram avaliados estatisticamente. Concluiu-se que a realização de teste de esforço em esteira ergométrica é útil para a determinação destes parâmetros e pôde-se observar as alterações que ocorrem nos valores durante o esforço físico intenso.

Palavras-chave: eqüino, esteira, exercício, hematologia.

Abstract

Thoroughbred horses are spread in Brazil and due to the lack of data concerning their exercise physiology, it is important to do exercise tests under some characteristic conditions in order to obtain precise and proper data as this horses are submitted to constant efforts. For many years, measures as PCV (packed cell volume) and hemoglobin concentration were widely used to evaluate athletic ability. Thus, twenty Thoroughbred racehorses in training at Jockey Club Brasileiro, with age ranging from 2 to 6 years old, males and females, submitted to effort tests on treadmill in order to evaluate hematological (RBC, WBC, PCV, total proteins and hemoglobin concentrations) values. The mean values varied at the studied times and they were statistically evaluated and correlated. It was concluded that the exercise tests on a treadmill are useful for the determination of this parameters and we can observe the alterations that occur in values during high intensity exercise.
\end{abstract}

Keywords: equine, treadmill, exercise, hematology.

\section{Introdução}

O cavalo (Equus caballus) encontra-se hoje amplamente difundido no mundo servindo ao homem não só para o lazer, mas também nos esportes e, dentre todos os que os envolvem, o turfe tem papel econômico de marcada importância. Dada a falta de dados sobre a hematologia destes animais durante esforço físico, decidiu-se coletar-lhes o sangue e observar o comportamento hematológico desde o início até o final do esforço.
Foram avaliados neste experimento: contagem total de hemácias, contagem total de leucócitos, hematócrito ou volume globular (VG), concentração de hemoglobina, volume globular médio (VGM), concentração de hemoglobina corpuscular média ( $\mathrm{CHCM}$ ) e proteínas totais plasmáticas.

O hemograma de repouso é bastante utilizado para detectar anormalidades que não são observadas clinicamente, estresse dos animais, sobrecarga de trabalho ou treinamento inadequado (Rose e Hodgson ${ }^{\mathrm{C}}$, 1994).

\footnotetext{
* Veterinária do Jockey Club Brasileiro.

** Prof ${ }^{a}$. Dr ${ }^{a}$. do Departamneto de Clínica e Patologia Clínica da Universidade Federal Fluminense.

*** Veterinária autônoma do Jockey Club Brasileiro.

**** Veterinário autônomo.

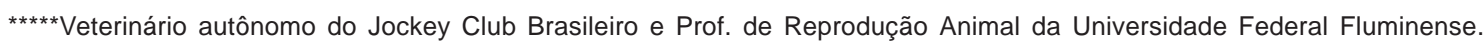


Ocorrem aumentos dos valores de hematimetria do cavalo de corrida de acordo com a intensidade do exercício a que são submetidos. Estes aumentos se devem à hemoconcentração e também devido à contração esplênica (o baço pode armazenar até $50 \%$ do total de células vermelhas), (Rose, s.d; Santos, 1960; Jain, 1993; Kingston e Bayly, 1998; Manohar et ali, 2001). Esses valores tendem a normalizar em, aproximadamente, 45 minutos após a atividade física (Santos, 1960).

De acordo com Rose (s.d.), a redistribuição do volume de sangue circulante que ocorre durante o exercício se constitui em importante adaptação para o trabalho muscular, pois ocorre dilatação dos vasos sanguíneos dos músculos, que passam a receber aproximadamente $70 \%$ do débito cardíaco, quando comparado aos $15 \%$ que recebem durante o repouso.

\section{VG:}

A medida do hematócrito $(\mathrm{Ht})$ serve como índice da capacidade de exercício, pois, o volume total de hemácias é determinante da capacidade de carrear oxigênio (Rose e Hodgson ${ }^{\mathrm{D}}$, 1994). O hematócrito aumenta linearmente com o aumento da intensidade do exercício, sendo que em cavalos PSI pode chegar a 61\% (Seeherman e Morris, 1990; Jain, 1993, Evans, 2000).

A contração esplênica do cavalo aproximadamente dobra o hematócrito com o consumo máximo de oxigênio (Evans, 2000).

Segundo Latimer e Mahaffey (1999), os valores normais de hematócrito estão entre 32 e $48 \%$.

\section{Hematimetria (RBC):}

O grande aumento de eritrócitos na circulação é vantajoso, pois ocorre aumento da capacidade de carrear oxigênio (Rose, s.d.; Snow e Mackenzie, 1977; Foreman, 1992).

O baço serve como reserva de eritrócitos e pode lançá-los na circulação quando há algum tipo de excitação (a simples venopunção por uma pessoa estranha pode excitar um cavalo) ou durante exercícios físicos (Foreman, 1992; Jain, 1993).

O aumento da capacidade de transporte de oxigênio associada à liberação de eritrócitos durante o exercício, isto é, maior concentração de hemoglobina, é um dos fatores mais importantes para a capacidade aeróbica alta dos cavalos. Entretanto, em determinado momento a capacidade de carrear oxigênio fica prejudicada pelo aumento da viscosidade do sangue com conseqüente redução da performance (Evans et al., 1993; Jain, 1993).

Os valores de normalidade, segundo Latimer e Mahafey (1999), são de 6-12 milhões/microlitro.

\section{Concentração de hemoglobina $(\mathrm{Hb})$ :}

A hemoglobina é uma proteína de estrutura quaternária encontrada nos eritrócitos que exerce duas principais funções: transportar oxigênio dos pulmões aos tecidos periféricos e transportar dióxido de carbono dos tecidos periféricos aos pulmões, para excreção (Mayes, 1998).

A quantidade de oxigênio que pode ser carreado pelo sangue está relacionado com o volume total de eritrócitos e concentração total de hemoglobina (Rose e Hodgson ${ }^{\mathrm{N}}$, 1994).
No exercício intenso uma maior quantidade de oxigênio deve ser liberado da hemoglobina para os tecidos (efeito Bohr) e isso ocorre porque a circulação de sangue para os tecidos aumenta causando leve queda da $\mathrm{pO}_{2}$ tecidual e liberando grandes quantidades de oxigênio para os tecidos (Guyton e Hall, 1996).

Os valores normais são de 10-16g/dl, de acordo com Latimer e Mahaffey (1999).

\section{Volume Corpuscular Médio (VCM) e Concentração de Hemoglobina Corpuscular Média (CHCM):}

Segundo Lassen e Swardson (1995), define-se VCM e CHCM como:

VCM: é o tamanho médio dos eritrócitos e é calculado através da seguinte fórmula:

$\mathrm{VCM}=\mathrm{PCV}(\%) / \mathrm{RBC}$ (em milhões $/ \mathrm{ml}) \times 10$

CHCM: é a medida da concentração da hemoglobina dos eritrócitos, ou seja, representa a quantidade de hemoglobina/ eritrócito. É calculada pela seguinte fórmula:

$\mathrm{CHCM}(\mathrm{g} / \mathrm{dl})=\mathrm{Hb}(\mathrm{g} / \mathrm{dl}) / \mathrm{PCV}(\%) \times 100$

Segundo Jain (1993), animais que vivem em altitudes elevadas apresentam maiores contagens do número total de eritrócitos, da concentração de hemoglobina e do volume globular quando comparados àqueles que vivem em lugares de baixas altitudes. E, ainda, segundo o mesmo autor, em adição às mudanças nos valores referentes às células vermelhas ocorre aumento do VCM e diminuição da CHCM após exercícios intensos.

Leucograma (WBC):

o leucograma varia com a intensidade e duração do exercício, podendo haver aumentos de 10 a $30 \%$, bem como com o grau de estresse a que o cavalo está submetido. O leucograma tem sido utilizado como indicador de estresse de treinamento em cavalos de corridas (Tyler-McGowan, 1999).

O exercício máximo provoca liberação de leucócitos sequestrados pelo baço e provenientes do pool marginal. Ocorre aumento do número de linfócitos e diminuição da relação neutrófilos/linfócitos. A linfocitose é transitória depois do exercício máximo e volta ao normal depois de algumas horas (Rose, s.d.; Jain 1993).

\section{Proteínas Totais Plasmáticas $(\mathrm{Pt})$ :}

o aumento da concentração de proteínas totais séricas durante o exercício de alta intensidade reflete o movimento compensatório de fluidos dos vasos para os tecidos, aumentando a viscosidade do plasma (Tyler-McGowan, 1999; Rose

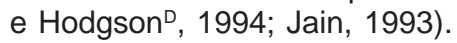

A extensão do deslocamento e perda de fluídos parece estar relacionada com a duração e intensidade do exercício. Muitas vezes, as alterações nos movimentos de fluidos podem retornar aos valores normais em até 30 minutos depois do exercício. A extensão de fluidos perdidos através do suor é mínima em muitos cavalos, representando perdas de 5 a 10 $\mathrm{kg}$ de peso corporal depois de uma corrida (ibid.). 


\section{Material e métodos}

Foram utilizados neste trabalho 20 animais da raça Puro-Sangue-Inglês (PSI), na faixa etária de 2 a 6 anos, machos e fêmeas, em fase de treinamento no Jockey Club Brasileiro.

Em todos os animais foram realizados exames clínicos rotineiros, em repouso, e avaliação do histórico do seu desempenho atlético. Todos os animais apresentaram boas condições físicas, sem quaisquer problemas aparentes.

Os animais foram submetidos a um período de adaptação ao exercício em esteira ergométrica GALLOPER 5500. O programa de adaptação levou de cinco a sete dias, consistindo desde o simples reconhecimento do animal à esteira até o galope confortável. Nesta fase foram registrados o batimento cardíaco e o comportamento dos animais nas diferentes velocidades a que foram submetidos. 0 programa de adaptação foi modificado ou ajustado às peculiaridades de cada animal.

Uma vez estando o animal apto a realizar o teste de esforço, seguiu-se o procedimento adiante descrito:

1) Foram realizadas coletas de amostras de sangue na cocheira, com o animal em repouso, em tubos de coleta a vácuo ${ }^{1}$ com EDTA, para análise hematológica. As amostras eram imediatamente armazenadas em recipiente isotérmico e levadas em seguida ao laboratório do Hospital Octávio Dupont, onde eram realizadas as análises.

2) A preparação do animal para a realização do teste de esforço consistiu de: a) tricotomia do local onde se situa topograficamente a veia jugular direita; b) rigorosa assepsia; c) anestesia do local da punção venosa com lidocaína $2 \%$ sem vasoconstritor; ${ }^{2}$ d) punção venosa e instalação de cateter ${ }^{3}$ e sistema extensor; e) fixação do cateter na pele com fio Vicryl 2.0; ${ }^{4}$ f) heparinização (solução de cloreto de sódio a $0,9 \%{ }^{5}$ e heparina sódica, ${ }^{6}$ do sistema coletor de amostras.

3) $O$ animal então era conduzido à esteira ergométrica, davase a inclinação de 6 graus da esteira em relação ao solo, para simular condições similares àquelas estabelecidas em condições de corridas, como compensar a ausência do peso do jóquei, e dava-se início ao exercício.

4) As velocidades impostas e amostras de sangue coletadas para as análises se deram da seguinte forma (Tabela 1):

\footnotetext{
1 Tubos para coleta de sangue a vácuo Vacutainer $\mathrm{BD}$

${ }^{2}$ Cloridrato de lidocaína 2\% sem vasoconstritor - Xilestein 2\% - Crystalia

${ }^{3}$ Cateter Intracath $12 \mathrm{G} \mathrm{BD}$

${ }^{4}$ Fio Vicryl 2.0 - Ethicon

${ }^{5}$ Solução de cloreto de sódio 0,9\% - Fresenius Kabi

${ }^{6}$ Heparina sódica - Liquemine - Roche $-25.000 \mathrm{UI}$
}

Tabela 1: Esquema representativo das velocidades $(\mathrm{m} / \mathrm{s})$ e coletas em cada momento do teste de esforço

\begin{tabular}{|c|c|c|c|c|c|}
\hline Exames Laboratoriais & RBC & $\mathrm{Ht}$ & $\mathrm{Hb}$ & $\mathbf{P t}$ & WBC \\
\hline Repouso & * & * & * & * & * \\
\hline $\begin{array}{c}\text { Passo } \\
(2 \mathrm{~m} / \mathrm{s}-2 \mathrm{~min} .)\end{array}$ & * & * & * & * & * \\
\hline $\begin{array}{c}\text { Trote } \\
(3 \mathrm{~m} / \mathrm{s}-4 \mathrm{~min} .)\end{array}$ & * & * & * & * & * \\
\hline $\begin{array}{c}\text { Galope } \\
(4 \mathrm{~m} / \mathrm{s}-1 \mathrm{~min} .)\end{array}$ & * & * & * & * & * \\
\hline $\begin{array}{c}\text { Galope } \\
(5 \mathrm{~m} / \mathrm{s}-1 \mathrm{~min} .)\end{array}$ & * & * & * & * & * \\
\hline $\begin{array}{c}\text { Galope } \\
(6 \mathrm{~m} / \mathrm{s}-1 \mathrm{~min} .)\end{array}$ & * & * & * & * & * \\
\hline $\begin{array}{c}\text { Galope } \\
(7 \mathrm{~m} / \mathrm{s}-1 \mathrm{~min} .)\end{array}$ & * & * & * & * & * \\
\hline $\begin{array}{c}\text { Galope } \\
(8 \mathrm{~m} / \mathrm{s}-1 \mathrm{~min} .)\end{array}$ & * & * & * & * & * \\
\hline $\begin{array}{c}\text { Galope } \\
(9 \mathrm{~m} / \mathrm{s}-1 \mathrm{~min} .)\end{array}$ & * & * & * & * & * \\
\hline $\begin{array}{c}\text { Galope } \\
(10 \mathrm{~m} / \mathrm{s} \text { ou maior }-1 \mathrm{~min} .)\end{array}$ & * & * & * & * & * \\
\hline Fim do Exercício & * & * & * & * & * \\
\hline 10 min. após & * & * & * & * & * \\
\hline
\end{tabular}

nde: RBC - contagem total de células vermelhas; Ht - hematócrito; Hb hemoglobina; Pt - proteína total; WBC - contagem total de células brancas.

Faltando 15 segundos para cada troca de velocidade, davase início à coleta da amostra. Primeiramente coletando-se $40 \mathrm{ml}$ que seriam descartados por estarem diluídos com a solução heparinizada e, posteriormente, coletando-se os $10 \mathrm{ml}$ da amostra a ser avaliada. Para isso foram utilizadas seringas de 60 e de $20 \mathrm{ml},{ }^{7}$ respectivamente. O sangue coletado nas seringas de $20 \mathrm{ml}$ era transferido para os tubos Vacutainer com EDTA. As seringas de $20 \mathrm{ml}$ foram desprezadas após serem utilizadas. Imediatamente após a coleta, o sistema de cateter e extensor eram heparinizados. O teste de esforço seguiu até a velocidade máxima que o animal conseguiu desenvolver, mesmo com todo incentivo da equipe. As velocidades máximas obtidas variaram de $10-12 \mathrm{~m} / \mathrm{s}$, sendo que um animal atingiu somente até $9 \mathrm{~m} / \mathrm{s}$.

Seguiu-se a coleta das amostras no momento 10 minutos após o exercício, com o animal ainda na esteira, em velocidade de passo ou já fora da esteira, mas caminhando na área próxima do exercício.

As amostras foram analisadas em Contador eletrônico de células CC-530 da CELM (RBC, Hb e WBC), centrífuga (Ht) e refratômetro $(\mathrm{Pt})$. VGM e CHCM foram calculados através de fórmulas já citadas anteriormente.

Em todos os momentos acompanhados, foram observadas e anotadas as freqüências cardíacas dos animais, sendo importante para este trabalho as freqüências nos instantes finais do teste de esforço, para determinação do seu término. A mínima freqüência observada na fase final do exercício foi de 205 bpm e a máxima foi de 237 bpm. A V ${ }_{200}$ (velocidade na qual o cavalo atinge 200 batimentos cardíacos por minuto) foi utilizada como base para o término do exercício.

\footnotetext{
7 Seringas de 10, 20 e $60 \mathrm{ml}$ Plastipak BD
} 
O tempo de permanência do animal na esteira durante o teste de esforço variou de 14 a 18 minutos, dependendo da velocidade atingida pelo animal no seu máximo esforço físico.

\section{Delineamento estatístico}

Os dados laboratoriais foram analisados estatisticamente por método não paramétrico de Wilcoxon, com nível de significância de $5 \%$ (Rodrigues, 1993).

\section{Resultados e discussão}

Tabela 2: VALORES DE HEMATIMETRIA (RBC) $\left(/ \mathrm{mm}^{3}\right)$ de eqüinos submetidos a teste de esforço em esteira ergométrica, em repouso (RBC-0); nas velocidades de: $2 \mathrm{~m} / \mathrm{s}$ (RBC-2); $3 \mathrm{~m} / \mathrm{s}$ (RBC-3); $4 \mathrm{~m} / \mathrm{s}$ (RBC-4:); 5m/s (RBC-5); 6m/s (RBC-6:);7m/s (RBC-7); 8m/s (RBC-8:); 9m/s (RBC-9:) ;10m/s (RBC10:); $11 \mathrm{~m} / \mathrm{s}$ (RBC-11); 12m/s (RBC-12:); no momento depois do exercício (RBC-D) e $10 \mathrm{~min}$. depois do exercício (RBC-10D)

\begin{tabular}{l|l|c|c|c|c} 
& $\mathbf{n}$ & MÉDIA & DESVIO-PADRÃO & VALOR MÍNIMO & VALOR MÁXIMO \\
\hline RBC-0 & 20 & 8.662 .000 & 662.567 & 7.580 .000 & 10.210 .000 \\
\hline RBC-2 & 20 & 10.603 .000 & 815.430 & 8.920 .000 & 12.210 .000 \\
\hline RBC-3 & 20 & 10.971 .500 & 734.390 & 9.900 .000 & 12.370 .000 \\
\hline RBC-4 & 20 & 11.196 .000 & 721.083 & 10.100 .000 & 12.780 .000 \\
\hline RBC-5 & 20 & 11.583 .000 & 711.929 & 10.570 .000 & 13.180 .000 \\
\hline RBC-6 & 20 & 11.791 .500 & 651.655 & 10.610 .000 & 13.190 .000 \\
\hline RBC-7 & 20 & 12.072 .500 & 644.889 & 10.990 .000 & 13.400 .000 \\
\hline RBC-8 & 20 & 12.441 .500 & 558.553 & 11.580 .000 & 13.580 .000 \\
\hline RBC-9 & 20 & 12.660 .000 & 635.916 & 11.380 .000 & 13.790 .000 \\
\hline RBC-10 & 17 & 12.898 .235 & 748.709 & 11.400 .000 & 14.210 .000 \\
\hline RBC-11 & 11 & 12.879 .091 & 1.326 .178 & 9.390 .000 & 14.410 .000 \\
\hline RBC-D & 3 & 13.113 .333 & 716.682 & 12.370 .000 & 13.800 .000 \\
\hline RBC-D10 & 20 & 12.859 .000 & 668.004 & 11.410 .000 & 14.210 .000 \\
\hline
\end{tabular}

$\mathrm{n}$ : número de animais avaliados.

Para todos os momentos houve diferença significativa $(p$ valor $<0,05)$ da contagem total de hemácias (RBC) com o momento anterior, com exceção de 10-11, 11-12 e 12-D.

Tabela 3: VALORES DE VOLUME GLOBULAR (VG) (\%) de eqüinos submetidos à teste de esforço em esteira ergométrica, em repouso (VG-0); nas velocidades de: $2 \mathrm{~m} / \mathrm{s}$ (VG-2); $3 \mathrm{~m} / \mathrm{s}$ (VG-3); $4 \mathrm{~m} /$ $\mathrm{s}(\mathrm{VG}-4:) ; 5 \mathrm{~m} / \mathrm{s}$ (VG-5); 6m/s (VG-6:); 7m/s (VG-7); 8m/s (VG-8:); 9m/s (VG-9:); 10m/s (VG-10:); $11 \mathrm{~m} / \mathrm{s}$ (VG-11); 12m/s (VG-12:); no momento depois do exercício (VG_D) e $10 \mathrm{~min}$. depois do exercício (VG-10D)

\begin{tabular}{l|l|c|c|c|c}
\hline & n & MÉDIA & DESVIO-PADRÃO & VALOR MÍNIMO & VALOR MÁXIMO \\
\hline VG-0 & 20 & 43.50 & 3.34 & 37.00 & 51.00 \\
\hline VG-2 & 20 & 53.25 & 3.89 & 46.00 & 61.00 \\
\hline VG-3 & 20 & 55.15 & 3.54 & 50.00 & 62.00 \\
\hline VG-4 & 20 & 56.25 & 3.47 & 50.00 & 64.00 \\
\hline VG-5 & 20 & 58.35 & 3.57 & 53.00 & 66.00 \\
\hline VG-6 & 20 & 58.90 & 3.32 & 53.00 & 66.00 \\
\hline VG-7 & 20 & 60.40 & 3.21 & 55.00 & 67.00 \\
\hline VG-8 & 20 & 62.00 & 3.07 & 56.00 & 69.00 \\
\hline VG-10 & 20 & 63.35 & 3.11 & 57.00 & 71.00 \\
\hline VG-11 & 17 & 64.64 & 3.70 & 57.00 & 72.00 \\
\hline VG-12 & 3 & 64.45 & 6.62 & 47.00 & 69.00 \\
\hline VG-D & 20 & 64.40 & 3.51 & 62.00 & 71.00 \\
\hline VG-D10 & 19 & 58.52 & 3.33 & 57.00 & 66.00 \\
\hline
\end{tabular}

$\mathrm{n}$ : número de animais avaliados. 
Para todos os momentos houve diferença significativa ( $p$ valor $<0,05$ ) do hematócrito (VG) com o momento anterior, com exceção de 10-11, 11-12 e 12-D.

Tabela 4: VALORES DE HEMOGLOBINA $(\mathrm{Hb})(\mathrm{g} / \mathrm{dL})$ de equinos submetidos à teste de esforço em esteira ergométrica, em repouso ( $\mathrm{Hb}-0)$; nas velocidades de: $2 \mathrm{~m} / \mathrm{s}(\mathrm{Hb}-2) ; 3 \mathrm{~m} / \mathrm{s}(\mathrm{Hb}-3) ; 4 \mathrm{~m} / \mathrm{s}(\mathrm{Hb}-4:)$; $5 \mathrm{~m} / \mathrm{s}(\mathrm{Hb}-5) ; 6 \mathrm{~m} / \mathrm{s}(\mathrm{Hb}-6:) ; 7 \mathrm{~m} / \mathrm{s}(\mathrm{Hb}-7) ; 8 \mathrm{~m} / \mathrm{s}(\mathrm{Hb}-8:) ; 9 \mathrm{~m} / \mathrm{s}(\mathrm{Hb}-9:) ; 10 \mathrm{~m} / \mathrm{s}(\mathrm{Hb}-10:) ; 11 \mathrm{~m} / \mathrm{s}(\mathrm{Hb}-11)$; 12m/s (Hb-12:); no momento depois do exercício (Hb-D) e $10 \mathrm{~min}$. depois do exercício (Hb-10D)

\begin{tabular}{l|c|c|c|c|c}
\hline & $\mathbf{n}$ & MÉDIA & DESVIO-PADRÃO & VALOR MÍNIMO & VALOR MÁXIMO \\
\hline $\mathbf{H b}-\mathbf{0}$ & 20 & 14.40 & 1.14 & 12.70 & 17.00 \\
\hline $\mathbf{H b}-\mathbf{2}$ & 20 & 17.67 & 1.47 & 14.30 & 20.10 \\
\hline $\mathbf{H b}-\mathbf{H}$ & 20 & 18.38 & 1.31 & 16.00 & 20.80 \\
\hline $\mathbf{H b}-\mathbf{4}$ & 20 & 18.76 & 1.24 & 16.70 & 21.10 \\
\hline $\mathbf{H b}-\mathbf{6}$ & 20 & 19.35 & 1.19 & 17.40 & 21.80 \\
\hline $\mathbf{H b}-\mathbf{H b}$ & 20 & 19.74 & 1.02 & 17.80 & 21.90 \\
\hline $\mathbf{H b}-\mathbf{9}$ & 20 & 20.21 & 1.02 & 18.40 & 22.10 \\
\hline $\mathbf{H b}-\mathbf{1 0}$ & 20 & 20.68 & 1.00 & 18.70 & 22.50 \\
\hline $\mathbf{H b}-\mathbf{1 1}$ & 17 & 21.58 & 1.09 & 18.80 & 23.00 \\
\hline $\mathbf{H b}-\mathbf{1 2}$ & 11 & 21.46 & 1.21 & 19.00 & 23.60 \\
\hline $\mathbf{H b}-\mathbf{D 1 0}$ & 3 & 22.03 & 2.12 & 15.80 & 23.80 \\
\hline
\end{tabular}

$\mathrm{n}$ : número de animais avaliados.

Para todos os momentos houve diferença significativa ( $\mathrm{p}$ valor $<0,05)$ da hemoglobina $(\mathrm{Hb})$ com o momento anterior, com exceção de 10-11, 11-12 e 12-D.

Tabela 5: VALORES DE LEUCOMETRIA TOTAL (WBC) $\left(/ \mathrm{mm}^{3}\right)$ de eqüinos submetidos à teste de esforço em esteira ergométrica, em repouso (WBC-0); nas velocidades de: $2 \mathrm{~m} / \mathrm{s}$ (WBC-2); 3m/s (WBC-3); 4m/s (WBC-4); $5 \mathrm{~m} / \mathrm{s}$ (WBC-5); 6m/s (WBC-6:); 7m/s (WBC-7); 8m/s (WBC-8:); 9m/s (WBC-9:); 10m/s (WBC10:); 11m/s (WBC-11); 12m/s (WBC-12:); no momento depois do exercício (WBC-D) e 10 min depois do exercício (WBC-10D).

\begin{tabular}{|c|c|c|c|c|c|}
\hline & $\mathbf{n}$ & MÉDIA & DESVIO-PADRÃO & VALOR MÍNIMO & VALOR MÁXIMO \\
\hline WBC-0 & 20 & 9.010 & 2.018 & 6.100 & 14.000 \\
\hline WBC-2 & 20 & 10.250 & 1.738 & 7.200 & 13.700 \\
\hline WBC-3 & 20 & 10.435 & 1.619 & 7.700 & 13.300 \\
\hline WBC-4 & 20 & 10.635 & 1.817 & 7.900 & 14.100 \\
\hline WBC-5 & 20 & 10.730 & 1.844 & 8.300 & 14.100 \\
\hline WBC-6 & 20 & 10.760 & 1.857 & 8.100 & 14.400 \\
\hline WBC-7 & 20 & 10.730 & 1.800 & 8.300 & 14.100 \\
\hline WBC-8 & 20 & 10.610 & 1.764 & 8.300 & 13.900 \\
\hline WBC-9 & 20 & 10625 & 1.775 & 8.500 & 14.100 \\
\hline WBC-10 & 17 & 10.423 & 1.630 & 8.100 & 13.800 \\
\hline WBC-11 & 11 & 10.172 & 2.400 & 5.800 & 14.100 \\
\hline WBC-12 & 3 & 11.300 & 3.251 & 8.900 & 15.000 \\
\hline WBC-D & 20 & 10.495 & 1.774 & 8.000 & 14.200 \\
\hline WBC-D10 & 19 & 9.552 & 1.917 & 6.000 & 13.600 \\
\hline
\end{tabular}

$\mathrm{n}$ : número de animais avaliados.

Para todos os momentos não houve diferença significativa ( $p$ valor $<0,05$ ) da contagem total de leucócitos (WBC) com o momento anterior, com exceção de 0-2 e D-D10. 
Tabela 6: VALORES DE PROTEÍNA TOTAL PLASMÁTICA (Pt) $(\mathrm{g} / \mathrm{dL})$ de equinos submetidos à teste de esforço em esteira ergométrica, em repouso (Pt-0); nas velocidades de: $2 \mathrm{~m} / \mathrm{s}(\mathrm{Pt}-2) ; 3 \mathrm{~m} / \mathrm{s}(\mathrm{Pt}-3) ; 4 \mathrm{~m} /$ $\mathrm{s}(\mathrm{Pt}-4:) ; 5 \mathrm{~m} / \mathrm{s}$ (Pt-5); 6m/s (Pt-6:); 7m/s (Pt-7); 8m/s (Pt-8:); 9m/s (Pt-9:); 10m/s (Pt-10); $11 \mathrm{~m} / \mathrm{s}$ (Pt-11); $12 \mathrm{~m} / \mathrm{s}$ (Pt-12:); no momento depois do exercício (Pt-D) e $10 \mathrm{~min}$. depois do exercício (Pt-10D)

\begin{tabular}{l|c|c|c|c|c}
\hline & $\mathbf{n}$ & MÉDIA & DESVIO-PADRÃO & VALOR MÍNIMO & VALOR MÁXIMO \\
\hline $\mathbf{P t - 0}$ & 20 & 6.35 & 0.59 & 5.20 & 7.80 \\
\hline $\mathbf{P t - 2}$ & 20 & 6.61 & 0.44 & 5.80 & 7.60 \\
\hline $\mathbf{P t - 3}$ & 20 & 6.81 & 0.47 & 6.00 & 8.00 \\
\hline $\mathbf{P t - 4}$ & 20 & 6.81 & 0.47 & 6.00 & 7.80 \\
\hline $\mathbf{P t - 5}$ & 20 & 7.01 & 0.59 & 6.20 & 8.00 \\
\hline $\mathbf{P t - 6}$ & 20 & 6.97 & 0.52 & 6.20 & 8.40 \\
\hline $\mathbf{P t - 7}$ & 20 & 7.06 & 0.49 & 6.20 & 8.20 \\
\hline $\mathbf{P t - 8}$ & 20 & 7.13 & 0.58 & 6.20 & 8.40 \\
\hline $\mathbf{P t - 9}$ & 20 & 7.28 & 0.50 & 6.40 & 8.20 \\
\hline $\mathbf{P t - 1 0}$ & 17 & 7.47 & 0.49 & 6.60 & 8.40 \\
\hline $\mathbf{P t - 1 1}$ & 11 & 7.23 & 1.57 & 2.80 & 8.60 \\
\hline $\mathbf{P t - 1 2}$ & 3 & 7.73 & 1.17 & 6.40 & 9.20 \\
\hline $\mathbf{P t - D}$ & 20 & 7.63 & 0.74 & 6.60 & 8.40 \\
\hline $\mathbf{P t - D 1 0}$ & 19 & 7.00 & 0.66 & 5.60 & \\
\hline
\end{tabular}

$\mathrm{n}$ : número de animais avaliados.

Para todos os momentos houve diferença significativa ( $p$ valor $<0,05)$ da proteína total $(\mathrm{Pt})$ com o momento anterior, com exceção de 3-4, 5-6, 10-11, 11-12 e 12-D.

Os processos induzidos pelo exercício são refletidos em alterações nos constituintes do sangue. Elevações do VG, da concentração de hemoglobina, na contagem total de hemácias e de leucócitos e na concentração de proteínas totais plasmáticas foram observadas neste trabalho.

Os valores referentes aos parâmetros hematológicos aumentaram conforme a intensidade do exercício, sendo que 10 minutos depois do término do teste houve reduções nos valores destes parâmetros. Segundo Seeherman e Morris (1990), alterações graduais ocorrem no período pós-exercício.

As elevações nos valores do eritrograma foram relacionadas com a contração esplênica devido à maior necessidade de oxigenação tissular (Young e Bermes, 1994). Rainger et al. (1995) também encontraram maiores concentrações de hemácias depois do exercício.

O aumento do VG e da concentração plasmática de proteínas, após exercício intenso, também foi observado por Evans et al. (1993), Seeherman e Morris (1990) e Snow e Mackenzie (1977). Esta elevação dos valores de proteínas está relacionado com o fato de que o exercício altera o volume plasmático devido ao deslocamento de líquido intravascular para o interstício, acrescido também da perda de líquido pela sudorese (Irjala e Gronroos, 1998).

Em estudo realizado por Tyler-McGowan (1999), comparando animais em diferentes fases de treinamento, observou que ocorre elevação na concentração de proteínas séricas, do VG, na contagem de hemácias e na concentração de hemoglobina depois do exercício. Mas não encontrou alterações na contagem total de leucócitos. Neste trabalho, porém, foram observadas alterações na contagem total de leucócitos e esta elevação foi relacionada com o estresse.

\section{Conclusão e sugestões}

O teste de esforço em esteira ergométrica permite a determinação de valores hematológicos, valores esses de extrema importância na avaliação da capacidade atlética desejável. Tal capacidade é compreendida como a integração dos vários sistemas corporais, os quais determinam a produção de energia despendida em uma disputa.

$\mathrm{Na}$ medida em que ocorre aumento da intensidade do exercício, vai se instalando um progressivo aumento da hemoconcentração.

As velocidades desenvolvidas na esteira podem ser semelhantes àquelas a que os animais desenvolvem nas corridas. Isto equivale a dizer que na esteira é possível desenvolver velocidade de rápida aceleração, a partir do momento em que está em inércia até uma velocidade de aproximadamente $15 \mathrm{~m} / \mathrm{s}$, para que seja possível simular condições similares àquelas estabelecidas em condições de corrida.

O acompanhamento da $\mathrm{V}_{200}$ (velocidade na qual o cavalo atinge 200 batimentos cardíacos por minuto) representa um dado clínico importante de avaliação de capacidade funcional, pois que é através do seu acompanhamento que se pode prever o momento no qual o animal apresentará fadiga. Nesta avaliação, deve-se afastar a possibilidade de ocorrência de ações sinérgicas do tipo ansiedade e estresse, ambos responsáveis pelo aumento da freqüência cardíaca.

Pode-se recomendar como válido o teste em esteira ergométrica, com a finalidade de padronizar os valores hematológicos nos cavalos de corridas, com vistas a uma avaliação mais confiável do seu condicionamento físico, levando-se ainda em consideração o fato de que o controle do monitoramento do animal costuma ser seguro e confiável durante as diferentes fases do teste. 


\section{Referências}

EVANS, D. L.; HARRIS, R. C.; SNOW, D. H. Correlation of Racing Performance with Blood Lactate and Heart Rate After Exercise in Thoroughbred Horses. Equine Veterinary Journal, v. 25, n. 5, p. 441445, 1993.

EVANS, D. L. Overview of Equine Exercise Physiology and Biochemistry. In: EVANS, D. L. (Ed.) Training and Fitness in Athletic Horses. Rural Industries Research and Development Corporation, 2000. cap. 2, p.10-32.

FOREMAN, J. H. Assessment of Performance Problems-Hematological and Endocrine Changes During Exercise - Assessment of Performance Problems. In: ROBINSON, Edward (Ed.) Current Therapy in Equine Medicine 3. W. B. Saunders Company, 1992. seção 19, p. 807-809.

GUYTON, A. C.; HALL, J. E. Regulation of Acid-Base Balance. In: Textbook of Medical Physiology. 9. ed. W. B. Saunders Company, 1996. 1148 p. cap. 30, p. 385-403.

IRJALA, K. M.; GRONROOS, P. Preanalytical and analytical factors affecting laboratory results. Annals of Medicine. V. 30, n. 3, 1998, p. 267-272.

MAYES, P. A. Bioenergética e Metabolismo de Carboidratos e Lipídeos. In: MURRAY, R. K.; GRANNER, D. K.; MAYES, P. A.; RODWELL, V.W. Harper. Bioquímica. 8. ed. São Paulo: Atheneu, 1998. Seção II, cap.12, p. 109-291.

JAIN, N. C. Examination of the Blood and Bone Marrow. In: Essentials of Veterinary Hematology. Lea \& Febiger, 1993. 417 p. cap. 1, p. 118.

Comparative Hematology of Common Domestic Animals. In: Essentials of Veterinary Hematology. Lea \& Febiger, 1993. 417 p. cap. 2, p. 19-53.

KINGSTON, J. K.; BAYLY, W. M. Effect of Exercise on Acid-Base Status of Horses. In: Veterinary Clinics of North America: Equine Practice. Fluids and Electrolytes in Athletic Horses. 1998. Abr, v. 14, n.1. p. 6173.

LASSEN. E. D.; SWARDSON, C. J. Hematology and Hemostasis in the Horse: Normal Functions and Common Abnormalities. In: Veterinary Clinics of North America: Equine Practice, Clinical Pathology. 1995. Dez, v. 11, n. 3, p. 351-389.
LATIMER, K. S.; MAHAFFEY, E. A. Ancillary Diagnostic Aids. In: COLAHAN, P. T.; MAYHEW, I. G.; MERRIT, A. M.; MOORE, J. N. Equine Medicine \& Surgery. 5. ed. Mosby, 1999. v. II, p. 1973-1980.

MANOHAR, M.; GOETZ, T. E.; HASSAN, A. S. Effect of Prior HighIntensity Exercise-Induced Arterial Hypoxemia in Thoroughbred Horses. Journal of Applied Physiology. v. 90, n. 6, p. 2371-2377, Jun, 2001.

RAINGER. J. E.; EVANS, D. L.; HODGSON, D. R.; ROSE, R. J. Distribution of Lactate in Plasma and Erythrocytes During and After Exercise in Horses. British Veterinary Journal, 151, p. 299-310, 1995.

RODRIGUES, P. C. Teste de Hipóteses. In: Bioestatística. 2. ed. Niterói: EdUFF, 1993. cap. 12, p. 79-88.

ROSE, R.J. Problems of the Performance and Endurance Horse - Poor Performance Syndrome: Investigation and Diagnostic Techniques. In: ROBINSON, E. (Ed.). Current Therapy in Equine Medicine 2. W. B. Saunders Company. seção 12, p. 469-474.

ROSE, R. J.; HODGSON, D. R. Structural Considerations in Equine Sports Medicine - Hematology and Biochemistry. In: The Athletic Horse. W. B. Saunders Company, 1994. 497 p, seção 1, cap. 5, p. 63-78.

SANTOS, R. Determinações Hematológicas em Cavalos PSI de Corrida, Antes e Depois do Exercício Muscular. 1960. Tese Apresentada à Cátedra de Patologia Geral e Semiologia da Escola Superior de Agricultura e Veterinária do Paraná. Universidade Federal do Paraná, Curitiba, 1960.

SEEHERMAN, H. J.; MORRIS, E.A. Application of a Standardised Treadmill Exercise Test for Clinical Evaluation of Fitness in 10 Thoroughbred Racehorses. Equine Veterinary Journal. n. 9, p. 26-34, Jun, 1990.

SNOW, D.H.; MACKENZIE, G. Some Metabolic Effects of Maximal Exercise in the Horse and Adaptations with Training. Equine Veterinary Journal. v. 9, n. 3, p. 134-140, 1977.

TYLER-McGOWAN, C. M.; GOLLAND, L. C.; EVANS, D. L.; HODGSON, D. R.; ROSE, R.J. Haematological and Biochemical Responses to Training and Overtraining. Equine Exercise Physiology 5. Equine Veterinary Journal. Suppl. 30, p. 621-625, 1999.

YOUNG.; BERMES. Specimen Collection and Processing - Sources of Biological Variation: Physiological Factors Affecting the Composition of Body Fluids. In: BURTIS, C. A.; ASHWOOD, E. R. Tietz Textbook of Clinical Chemistry. 2. ed. W. B. Saunders Company, 1994. cap. 2, p. 58-101. 